\title{
Dynamics of water confined in self-assembled monoglyceride-water-oil phases
}

\author{
Wolfgang Wachter, ${ }^{a}$ Gregor Trimmel, ${ }^{b}$ Richard Buchner ${ }^{c}$ and Otto Glatter ${ }^{* a}$ \\ Received 15th July 2010, Accepted 16th September 2010 \\ DOI: 10.1039/c0sm00681e
}

The dynamics of water confined in various self-assembled liquid-crystalline phases of the system Dimodan $\mathrm{U} / \mathrm{J}-R(+)$-limonene-water have been investigated by dielectric relaxation spectroscopy (DRS) over a wide range of frequencies $(0.2 \leq \nu / \mathrm{GHz} \leq 50)$. For all phases, two water-related Debye processes were detected, one slightly slower, the other considerably slower, than bulk water. Besides these bulk-like and slow-water relaxations, a further fraction of water does not appear in the dielectric spectra due to strong interactions with the interface (bound water). Increasing the water content of the inverse hexagonal $\left(\mathrm{H}_{\mathrm{II}}\right)$ phase leads to an increase in both bulk-like and bound water, whereas the amount of slow water remains constant. Furthermore, the bulk-like water relaxation times accelerate until they reach the pure-water value when approaching the saturation line. This finding is supported by differential scanning calorimetry (DSC), which also confirms the presence of three types of water in liquid-crystalline confinement.

\section{Introduction}

Inverse liquid-crystalline phases are formed by some amphiphiles, such as unsaturated monoglycerides, when in contact with water. Due to their unique properties they are a promising class of substances for both fundamental research and industrial use because they co-exist with an excess-water phase. This means they can be dispersed to form submicrometre-size particles. ${ }^{1-3}$ In the case of unsaturated monoglycerides, ${ }^{4}$ dispersion in water may give rise to particles exhibiting a number of different internal structures, ${ }^{5}$ such as emulsified microemulsions, cubosomes and hexosomes; the latter refer to the bicontinuous cubic and hexagonal dispersions found by Larsson et al. ${ }^{1 a, b}$ As a generalization, this entire 'family' of particles has been named ISAsomes (internally self-assembled particles). ${ }^{2}$ Many applications, especially in the fields of pharmaceutics, cosmetics or food industry, use ISAsomes as carriers for the incorporation and controlled release of guest molecules. ${ }^{6}$

In this context, understanding the properties of the water network confined inside the liquid-crystalline or microemulsion bulk phases is essential. The physico-chemical properties of bulk water, which exhibit a number of peculiarities mainly caused by the extraordinarily strong $\mathrm{H}$-bond network, are well known. ${ }^{7,8}$ However, it is at least questionable to assume that water reservoirs confined in narrow cavities should behave exactly like bulk water. In contrast, a number of studies suggest that geometrical confinement has a significant influence on the dynamics of water. ${ }^{9}$ Indeed, the dynamic, but also some thermodynamic properties of the water molecules situated directly at the interface (so-called bound water) deviate considerably from what is

${ }^{a}$ Department of Chemistry, Karl-Franzens-University Graz, Heinrichstraße 28, 8010 Graz, Austria

${ }^{b}$ Institute for Chemistry and Technology of Materials (ICTM), Graz University of Technology, Stremayrgasse 9, 8010 Graz, Austria

'Institute of Physical and Theoretical Chemistry, Regensburg University, 93040 Regensburg, Germany known for bulk water. ${ }^{10-13}$ Hence, the distinction between bound and bulk water is important for a better understanding not only of interactions between self-assembled amphiphiles and water, but also of biochemical processes ${ }^{13}$ Furthermore, the distinction also plays an important role in a number of industrial applications, where the evaporation rates of water confined in a microstructure are decisive parameters. ${ }^{14}$

Dielectric relaxation spectroscopy (DRS), which measures the complex dielectric response of a sample to an applied oscillating electric field as a function of the field frequency, is an efficient tool to study the dynamics of liquids exhibiting a permanent dipole moment. Thanks to its sensitivity to the collective modes of hydrogen-bonded systems and the reorientation of transient dipolar aggregates, DRS is an excellent complement to classical spectroscopic techniques such as IR, Raman or NMR spectroscopy, which are only able to monitor molecular dynamics and short-range interactions. In contrast, DRS sheds light on the dynamics of cooperative processes and their structural consequences. ${ }^{15,16}$ Hence, the method is particularly suitable for the investigation of water under confinement, especially since it has previously revealed some characteristic relaxation processes caused by hydrate water molecules surrounding the interfaces ${ }^{17}$ and micelles ${ }^{18}$ of various surfactants.

This contribution presents a DRS study covering inverse bicontinuous cubic $(P n 3 m)$, lamellar $\left(\mathrm{L}_{\alpha}\right)$ and inverse hexagonal $\left(\mathrm{H}_{\mathrm{II}}\right)$ phases in the systems Dimodan U/water, and Dimodan U/ $R(+)$-limonene/water, respectively. It will be shown that the dynamics of a large fraction of the water confined in these inverse liquid-crystalline structures are considerably slowed down due to interactions with the interface. However, the remaining water, situated further away from the interface, is only slightly affected and even adopts the characteristic bulk-water relaxation time when approaching the saturation line in the $\mathrm{H}_{\mathrm{II}}$ phase. This finding, which is supported by differential scanning calorimetry (DSC) data, is of special interest, since the internal structure of ISAsomes corresponds to that of bulk phases near the saturation line. 


\section{Experimental section}

\subsection{Materials and sample preparation}

All samples were prepared from distilled water, $R(+)$-limonene (Fluka, Switzerland, $>96 \%$, referred to as 'oil' in the following), and Dimodan U/J (DANISCO A/S, Denmark, abbreviated as 'DU' in the following); the latter contains $96 \%$ distilled monoglycerides, of which $62 \%$ are linoleate. Binary DU/water mixtures were prepared according to a previously published phase diagram. ${ }^{19}$ For the ternary DU/oil/water mixtures, the phase diagram of Fig. 1 (ref. 5) was used, where the DU/oil ratio is given by the parameter $\delta$ :

$$
\delta=\frac{m_{\mathrm{DU}}}{m_{\mathrm{DU}}+m_{\mathrm{oil}}}
$$

All samples investigated in this work were prepared gravimetrically without buoyancy correction; their compositions are given in Tables 1 and 2 .

After weighing DU, oil and water into Pyrex tubes, the mixtures were heated several times for a few seconds using a Bunsen burner with intermittent vigorous homogenization using a vortex. They were then allowed to equilibrate at room temperature for at least one week.

\subsection{Dielectric relaxation spectroscopy}

Dielectric spectra were recorded at $0.2 \leq \nu / \mathrm{GHz} \leq 50$ at Regensburg using two reflection probe heads, covering 0.2-20 $\mathrm{GHz}$ and $1-50 \mathrm{GHz}$, in conjunction with an Agilent E8364B vector network analyzer (VNA) and a corresponding ECal module. The instrument was calibrated with air, mercury and $N, N$-dimethylacetamide (DMA) following a previously published method. ${ }^{20}$ All VNA spectra were recorded at least twice using independent calibrations. The temperature was controlled to at least $\pm 0.04 \mathrm{~K}$. Typical spectra and corresponding fits (see below) are shown in Fig. 2-4; while all fitting parameters are given in Tables 1 and 2.

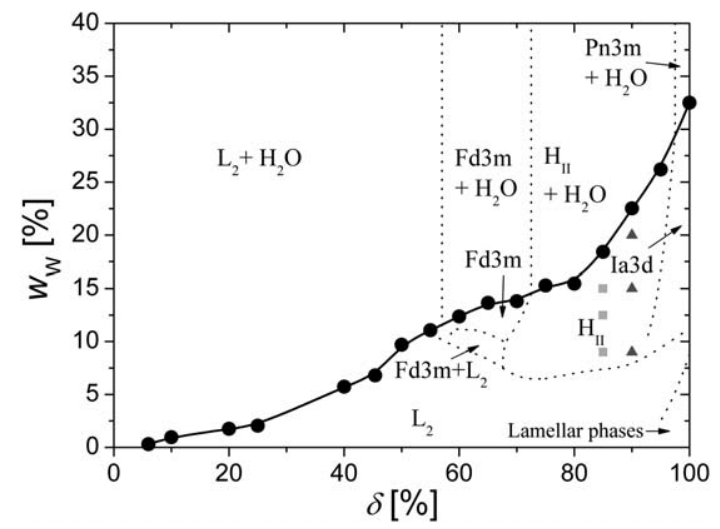

Fig. 1 Phase diagram of the ternary system DU/R(+)-limonene/ $\mathrm{H}_{2} \mathrm{O}$ at $25^{\circ} \mathrm{C}$ showing the maximum solubilization line of water $(\bullet$, data taken from ref. 5) and the $\mathrm{H}_{\mathrm{II}}$ samples investigated in this work ( $\mathbf{\square}: \delta=0.85$; $\boldsymbol{\Delta}: \delta=0.90)$.
Table 1 Phase, water mass fraction, $w_{\mathrm{W}}$, temperature, $\vartheta$, and parameters of the $3 \mathrm{D}$ model (limiting permittivities $\varepsilon_{\mathrm{s}}, \varepsilon_{2}, \varepsilon_{3}, \varepsilon_{\infty}$; relaxation times $\tau_{1}, \tau_{2}, \tau_{3}$; reduced error function, $\chi_{r}^{2}$ ) for the binary $\mathrm{DU} / \mathrm{H}_{2} \mathrm{O}$ samples investigated in this work. The parameter followed by the letter " $F$ " was not adjusted in the fitting procedure

\begin{tabular}{llllllllllll}
\hline Phase & $w_{\mathrm{W}}$ & $\vartheta /{ }^{\circ} \mathrm{C}$ & $\varepsilon_{\mathrm{s}}$ & $\tau_{1} / \mathrm{ps}$ & $\varepsilon_{2}$ & $\tau_{2} / \mathrm{ps}$ & $\varepsilon_{3}$ & $\tau_{3} / \mathrm{ps}$ & $\varepsilon_{\infty}$ & $\chi^{2}{ }_{\mathrm{r}}$ \\
\hline $\mathrm{L}_{\alpha}$ & 0.1287 & 25 & 8.78 & $217 \mathrm{~F}$ & 6.91 & 44.4 & 5.16 & 11.1 & 3.11 & 0.0012 \\
$\mathrm{~L}_{\alpha}$ & 0.1500 & 25 & 9.85 & 217 & 8.25 & 58.2 & 6.24 & 13.2 & 3.31 & 0.0016 \\
Pn3m & 0.2492 & 35 & 14.49 & 115 & 12.29 & 28.8 & 9.37 & 7.09 & 4.01 & 0.0044 \\
Pn3m & 0.3006 & 35 & 15.05 & 116 & 12.75 & 26.6 & 9.12 & 6.82 & 4.01 & 0.0058 \\
Pn3m & 0.3305 & 35 & 16.06 & 123 & 14.02 & 30.5 & 11.04 & 7.08 & 4.11 & 0.0043
\end{tabular}

Table 2 DU/oil mass ratio, $\delta$, water mass fraction, $w_{\mathrm{W}}$, and parameters of the 3D model (limiting permittivities $\varepsilon_{\mathrm{s}}, \varepsilon_{2}, \varepsilon_{3}, \varepsilon_{\infty}$; relaxation times $\tau_{1}$, $\tau_{2}, \tau_{3}$; reduced error function, $\chi_{r}^{2}$ ) of the ternary DU/R(+)-limonene/ $\mathrm{H}_{2} \mathrm{O}$ samples $\left(\mathrm{H}_{\mathrm{II}}\right.$ phase $)$ investigated in this work at $25^{\circ} \mathrm{C}$

\begin{tabular}{llllllllll}
\hline$\delta$ & $w_{\mathrm{W}}$ & $\varepsilon_{\mathrm{s}}$ & $\tau_{1} / \mathrm{ps}$ & $\varepsilon_{2}$ & $\tau_{2} / \mathrm{ps}$ & $\varepsilon_{3}$ & $\tau_{3} / \mathrm{ps}$ & $\varepsilon_{\infty}$ & $\chi^{2}{ }_{\mathrm{r}}$ \\
\hline 0.7006 & 0.0855 & 5.88 & $250 \mathrm{~F}$ & 5.17 & 41.7 & 4.18 & 11.7 & 3.26 & 0.0045 \\
0.7493 & 0.1248 & 7.10 & 520 & 6.41 & 57.8 & 5.05 & 8.98 & 3.28 & 0.0036 \\
0.8001 & 0.1246 & 6.53 & 249 & 5.86 & 35.8 & 4.28 & 7.67 & 3.19 & 0.0078 \\
0.8499 & 0.0899 & 6.61 & 341 & 5.73 & 58.7 & 4.31 & 9.33 & 3.20 & 0.0025 \\
0.8504 & 0.1250 & 7.24 & 270 & 6.32 & 58.0 & 4.84 & 9.60 & 3.46 & 0.0090 \\
0.8495 & 0.1505 & 7.68 & 401 & 6.98 & 60.4 & 5.52 & 8.94 & 3.58 & 0.0048 \\
0.8999 & 0.0910 & 7.19 & 291 & 5.96 & 66.7 & 4.41 & 10.4 & 3.21 & 0.0076 \\
0.8998 & 0.1527 & 8.03 & 435 & 7.26 & 58.9 & 5.38 & 8.89 & 3.57 & 0.0101 \\
0.9004 & 0.1997 & 9.10 & 446 & 8.30 & 58.2 & 6.65 & 8.21 & 3.98 & 0.0083 \\
\hline
\end{tabular}
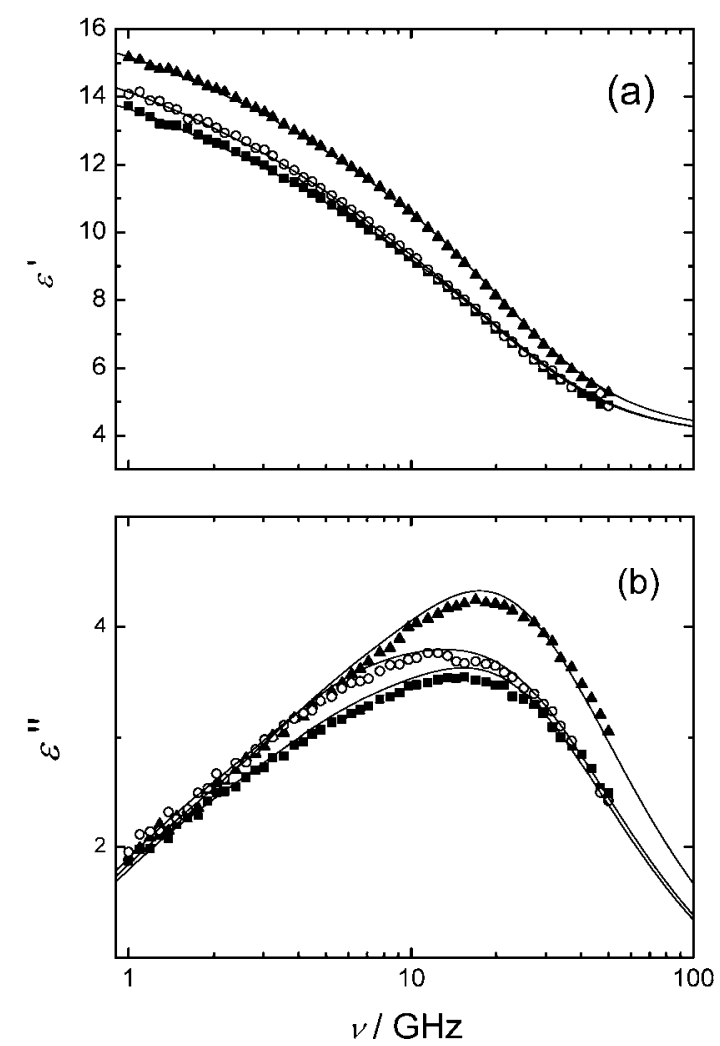

Fig. 2 Spectra of (a) dielectric permittivity, $\varepsilon^{\prime}(\nu)$ and (b) dielectric loss, $\varepsilon^{\prime \prime}(\nu)$, for DU/ $\mathrm{H}_{2} \mathrm{O}(P n 3 m$ phase $)$ at $35{ }^{\circ} \mathrm{C}$; water mass fractions, $w_{\mathrm{W}}=$ $0.25,0.30$, and 0.33 (bottom to top). 


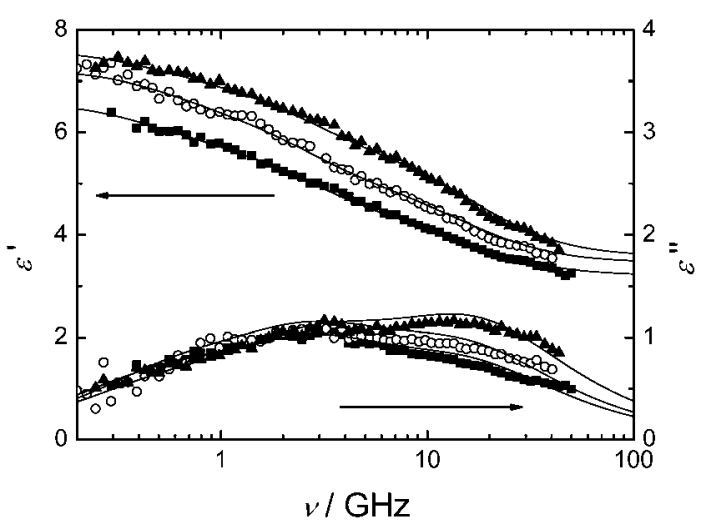

Fig. 3 Spectra of dielectric permittivity, $\varepsilon^{\prime}(\nu)$, and dielectric loss, $\varepsilon^{\prime \prime}(\nu)$, for DU/R(+)-limonene $/ \mathrm{H}_{2} \mathrm{O}\left(\mathrm{H}_{\mathrm{II}}\right.$ phase, $\left.\delta=0.85\right)$ at $25^{\circ} \mathrm{C}$; water mass fractions, $w_{\mathrm{W}}=0.09,0.125$, and 0.15 (bottom to top).

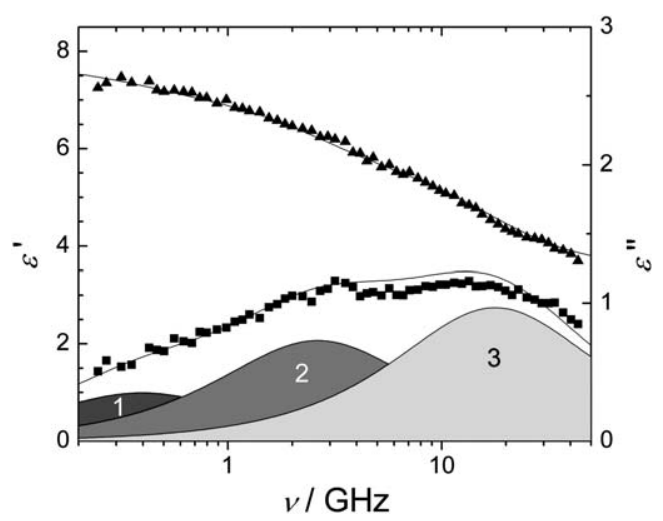

Fig. 4 Dielectric permittivity $(\Delta)$ and dielectric loss $(\boldsymbol{\square})$ spectrum of $\mathrm{DU} / R(+)$-limonene $/ \mathrm{H}_{2} \mathrm{O}\left(\mathrm{H}_{\mathrm{II}}\right.$ phase, $\left.\delta=0.85, w_{\mathrm{W}}=0.15\right)$ at $25^{\circ} \mathrm{C}$. Experimental data are described by a superposition of three Debye processes (3D model, eqn (3), solid line).

\subsection{Differential scanning calorimetry}

Calorimetric measurements were carried out using a PerkinElmer Pyris Diamond differential scanning calorimeter (DSC) equipped with a Perkin-Elmer CCA7 cooling system. The sample ( $18 \mathrm{mg}$ ) was weighed into standard $50 \mu \mathrm{L}$ aluminium pans and immediately sealed by a press. The samples were cooled using liquid nitrogen with a flow rate of $20 \mathrm{~mL} \mathrm{~min}{ }^{-1}$ from $30{ }^{\circ} \mathrm{C}$ to $-50{ }^{\circ} \mathrm{C}$ at $60 \mathrm{~K} \mathrm{~min}^{-1}$. Each sample was kept at $-50{ }^{\circ} \mathrm{C}$ for 30 min and was then heated to $10{ }^{\circ} \mathrm{C}$ at a rate of $1 \mathrm{~K} \mathrm{~min}^{-1}$. An unloaded pan served as a reference.

\section{Data analysis}

\subsection{Principles and models}

For a liquid of dc conductivity $\kappa$, DRS determines the relative dielectric permittivity, $\varepsilon^{\prime}(\nu)$, and the total loss, $\eta^{\prime \prime}(\nu)$, which is related to the dielectric loss $\varepsilon^{\prime \prime}(\nu)$ (see eqn (2)):

$$
\eta^{\prime \prime}(\nu)=\varepsilon^{\prime \prime}(\nu)+\kappa /\left(2 \pi \nu \varepsilon_{0}\right)
$$

In eqn (2) $\varepsilon_{0}$ is the vacuum permittivity. As no ionic species are present in the samples investigated in this work the dc conductivity is negligible, and thus we can assume $\eta^{\prime \prime}(\nu) \approx \varepsilon^{\prime \prime}(\nu)$. Provided the spectra of at least two independent calibrations recorded with the same probe head were reproducible, they were averaged, and the results of the two different probe heads were combined. As can be seen from Fig. 3 there was, in general, a seamless fit between the data points. Only for the very viscous Pn3m samples, we had to limit the data analysis to the frequency range $1 \leq \nu / \mathrm{GHz} \leq 50$, as the quality of the low-frequency data was not satisfactory.

The fitting of dielectric relaxation spectra requires care; our general approach to this problem has been outlined in detail previously, ${ }^{21,22}$ and thus only a brief description of the procedures is given here. The combined $\hat{\varepsilon}(\nu)$ data were analyzed by simultaneously fitting the in-phase $\left(\varepsilon^{\prime}(\nu)\right.$, see for example Fig. 2a) and the $90^{\circ}$ out-of-phase $\left(\varepsilon^{\prime \prime}(\nu)\right.$, Fig. $\left.2 \mathrm{~b}\right)$ components to various relaxation models consisting of $n$ distinguishable relaxation processes using a Levenberg-Marquardt algorithm.

Although many other models were investigated, it was found that the DRS data for the present systems were best described by a superposition of three Debye processes (the 3D model) (see eqn (3)):

$$
\widehat{\varepsilon}(\nu)=\varepsilon_{\infty}+\frac{S_{1}}{1+\left(i 2 \pi \nu \tau_{1}\right)}+\frac{S_{2}}{1+\left(i 2 \pi \nu \tau_{2}\right)}+\frac{S_{3}}{1+\left(i 2 \pi \nu \tau_{3}\right)}
$$

In eqn (3), $\varepsilon_{\infty}$ is the infinite-frequency permittivity and $\tau_{j}$ is the relaxation time for the $j^{\text {th }}$ dispersion step; the relaxation amplitudes $S_{j}$ are obtained from the limiting permittivities $\varepsilon_{j}$ of the individual relaxations as $S_{1}=\varepsilon-\varepsilon_{2}, S_{2}=\varepsilon_{2}-\varepsilon_{3}$, and $S_{3}=\varepsilon_{3}-$ $\varepsilon_{\infty}$ with $\varepsilon$ being the static permittivity of the sample. The parameters produced from the $3 \mathrm{D}$ model are given in Tables 1 and 2. As well as providing a set of self-consistent limiting permittivities and relaxation times for each dispersion step, the fitting model used gave smaller values of the reduced error function, $\chi_{r}^{2},{ }^{21}$ than any of the other models tried. In particular, no improvements could be achieved when replacing one of the Debye equations by a Cole-Cole or Cole-Davidson equation. ${ }^{15}$ On the other hand, our attempts to describe the present data using only two relaxation processes did not yield physically meaningful results.

\subsection{Process assignment}

The relaxation time of the highest-frequency process $3\left(\tau_{3} \approx 10\right.$ ps) is very similar to that of the cooperative relaxation of the $\mathrm{H}$ bond network observed in bulk water $\left(\tau_{\text {bulk }} \approx 8.32 \mathrm{ps}\right){ }^{8,23}$ Thus, this process can be attributed with confidence to the bulk-like water in the system.

Process 2 can also be ascribed to water, although its relaxation times are clearly higher $\left(25 \leq \tau_{2} / \mathrm{ps} \leq 60\right)$. Water relaxation on a similar timescale (so-called slow water) has previously been reported for a large number of aqueous electrolyte solutions containing hydrophobic or hydrophilic interfaces..$^{15,17,18,21,24}$ For example, in the case of large hydrophobic ions such as tetra- $n$ alkylammonium halides, ${ }^{18,21}$ or tetraphenylphosphonium and tetraphenylborate, ${ }^{17}$ the slow water relaxation has been ascribed to the water molecules surrounding the hydrophobic interfaces (so-called hydrophobic hydration). It is assumed that the reduced 
dynamics of these molecules are combinations of their restricted configurational space and screening effects. ${ }^{15}$

On the other hand, slow water has also been observed for aqueous solutions of rubidium fluoride ${ }^{24 a}$ and sodium formate and acetate ${ }^{24 b}$ in these cases, the slow water dynamics were assigned to $\mathrm{H}_{2} \mathrm{O}$ molecules hydrating the anion via $\mathrm{H}$-bonds. For the slow-water relaxation observed for $\mathrm{Na}^{+}$salts of the dicarboxylates malonate and succinate, ${ }^{24 c}$ contributions from both hydrophobic and hydrophilic hydration are likely. ${ }^{15}$

Given that a DU molecule features both a hydrophobic chain and a large hydrophilic head group, it is not surprising to observe slow water in the present system. Thus, process 2 may be explained by hydrophobic hydration of the hydrocarbon chains of the amphiphile, and/or can be ascribed to those water molecules situated close to the hydrophilic DU head groups. These two possible interpretations will be discussed in detail in Section 4

Other explanations for process 2 are difficult to conceive. Since test measurements of pure DU, pure limonene and of a $1: 1$ mixture of both components did not show any relaxation in this frequency range, it has to be concluded that process 2 is linked to the presence of water in the system. However, for aqueous systems, the only relaxations occurring on this timescale that are not water-related are due to ion pairs formed by electrolytes, which are absent in the present samples.

Process $1\left(120 \leq \tau_{1} / \mathrm{ps} \leq 400\right)$ is clearly outside the range of possible water relaxations and can most probably be explained by the uncorrelated movements of the hydrated dipolar head groups of the amphiphile: ${ }^{25}$ each DU head group features a small dipole moment. On average, due to the geometry of the inverse liquid-crystalline structures, a mutual cancellation of these single dipole moments can be assumed. However, every single DU head group has a certain degree of mobility in aqueous solution. As these head group rearrangements are statistical processes, they are not correlated over the entire liquid-crystalline structure. Thus, at a certain point of time, the charge distribution is not symmetrical any more, giving rise to a total dipole moment.

A similar relaxation has been observed for didodecyldimethylammonium bromide molecules at the interface of a W/O microemulsion. ${ }^{26}$ However, as process 1 is situated at the lowfrequency limit of our measurements, it cannot be excluded that it also contains a contribution from another relaxation occurring at lower frequencies. Therefore, we refrain from a quantitative discussion of the relaxation amplitudes $S_{1}$ and the relaxation times $\tau_{1}$.

\section{Results and discussion}

\subsection{Bulk, slow and bound water}

Before discussing the present data, it is necessary to briefly review existing knowledge about the different types of water behavior that can be observed with DRS.

The dielectric spectrum of pure water is dominated by a relaxation centered at $\sim 18 \mathrm{GHz}$, which describes the cooperative relaxation of the $\mathrm{H}$-bond network of bulk water. Its relaxation time $\left(\tau_{\text {bulk }} \approx 8.32 \mathrm{ps}\right)^{23}$ is interpreted as the 'dwelling time'; i.e., the time it takes until all $\mathrm{H}$-bonds except one of a water molecule are broken so that it can freely rotate. ${ }^{27,28}$
If considerable amounts of hydrophobic interfaces are present in the system (e.g. hydrophobic ions, or surfactants in micellar systems) a second water process arises that features a slower relaxation time (so-called slow water). ${ }^{18,21,24 c}$ This phenomenon can be understood on the following grounds: it is generally accepted that cooperative water dynamics are governed by the presence of so-called "fifth neighbors", i.e. water molecules coming close to a central water molecule, but not yet participating in its initial H-bond network. These fifth neighbors interact with the H-bond network, and thus modify the energy of the bound central water molecule, which in turn may evoke the breaking of H-bonds. Furthermore, the fifth neighbors offer an alternative to the existing $\mathrm{H}$-bond configuration, as they may act as new H-bond donors or acceptors; thus, their presence 'catalyzes' H-bond relaxation. However, a hydrophobic interface represents a steric obstacle to a fifth-neighbor water molecule; thus, the nearby water molecules are shielded from fifth neighbors' attacks; this can give rise to longer dwelling times (i.e. longer relaxation times) of a given $\mathrm{H}$-bond configuration.

Additionally, water molecules surrounding ions or other hydrophilic interfaces are, in general, strongly bound to these interfaces. In the case of $\mathrm{F}^{-}$or carboxylate groups, strong hydrogen-bond interactions cause retardation of the water dynamics. ${ }^{15,24 a, b}$ Coincidentally, this relaxation time overlaps with that of the $\mathrm{H}_{2} \mathrm{O}$ molecules surrounding hydrophobic moieties. ${ }^{15}$ As a result, the appearance of slow water could be due to either hydrophobic or hydrophilic hydration phenomena.

However, most ions or hydrophilic interfaces evoke such strong hydrogen-bond interactions with the nearby water molecules that the latter cannot rotate any more on the DRS timescale (irrotationally bound) and thus, they disappear from the dielectric spectrum. ${ }^{28}$ Although not directly visible in the dielectric spectrum the amount of such bound water can be calculated, provided the total water concentration of the investigated system is known (see below).

\subsection{Binary DU/water mixtures}

In the first part of this study, we started with the simplest possible system; binary DU/water mixtures; both the inverse bicontinuous cubic $P n 3 m$ and the lamellar $\mathrm{L}_{\alpha}$ phases were investigated. For both phases, an increase in the total amplitude, $S_{2}+S_{3}$, of the two water-related processes, $S_{2}$ and $S_{3}$, can be observed when increasing the water content. In contrast, $S_{1}$ does not show any significant changes (Fig. 5). In principle, it is possible to convert these amplitudes into the apparent molar concentrations $c^{\text {ap }}$ of the related species using the generalized Cavell equation: ${ }^{29}$

$$
c_{i}^{\mathrm{ap}}=\frac{3\left(\varepsilon+(1-\varepsilon) A_{i}\right)}{\varepsilon} \frac{k_{\mathrm{B}} T \varepsilon_{0}}{N_{\mathrm{A}}} \frac{\left(1-\alpha_{i} f_{i}\right)^{2}}{g_{i} \mu_{i}^{2}} S_{i}
$$

In eqn (4), $\varepsilon=\varepsilon^{\prime}(\nu \rightarrow 0)$ describes the static solution permittivity, $\varepsilon_{0}$ the vacuum permittivity, $N_{\mathrm{A}}$ and $k_{\mathrm{B}}$ are the Avogadro and Boltzmann constants, respectively, $T$ is the temperature, and $\mu_{i}$ and $\alpha_{i}$ are the dipole moment and polarizability of the relaxing species. The geometrical factors $A_{i}$ and $f_{i}$ were determined as described elsewhere. ${ }^{29}$

However, the values of the empirical orientational correlation factor $g_{i}$, which describes the orientation of single water dipoles relative to each other (similar to the theoretically based 


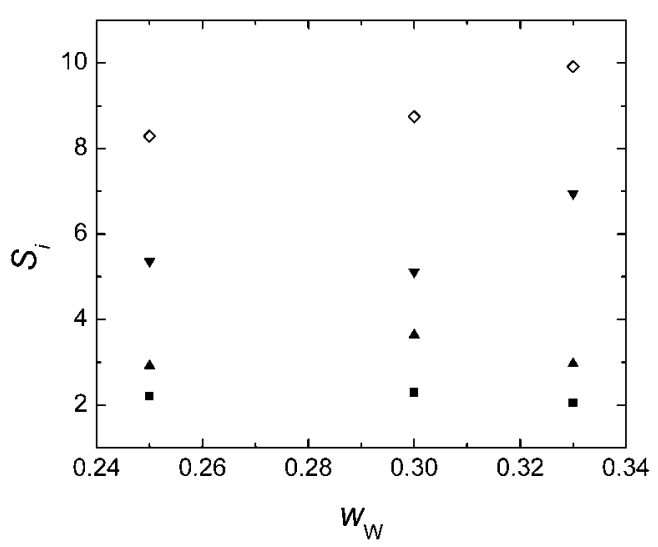

Fig. 5 Variation of relaxation amplitudes, $S_{i}$, of DU/ $\mathrm{H}_{2} \mathrm{O}\left(P n 3 m, 35^{\circ} \mathrm{C}\right)$ with water content, $w_{\mathrm{W}}: S_{1}(\boldsymbol{\square}), S_{2}(\boldsymbol{\nabla}), S_{3}(\boldsymbol{\Delta})$ and $S_{2}+S_{3}(\diamond)$. Similar results were obtained for $\mathrm{L}_{\alpha}$.

Kirkwood factor for systems exhibiting a single relaxation), are unknown for water confined in liquid-crystalline phases. It is well known that considerable dipole-dipole correlations are present in the tetrahedral network of bulk water, which leads to a parallel alignment of the water molecules, and thus to a Kirkwood factor of $g \approx 2.68 .{ }^{8}$ However, $g$ is highly susceptible to modifications of the water structure. For example, aqueous solutions of electrolytes featuring large ions like $\mathrm{NaBPh}_{4}$ can show a significant decrease in $g .{ }^{17}$ Therefore, as we are unaware of the correct value of $g$, we can only assume two limiting cases: the bulk-water value of $g=2.68$ and a completely statistical orientation of the water dipoles $(g=1)$.

Following this path and adding the concentrations of processes 2 and $3, c_{2}+c_{3}$, the apparent water concentration, $c_{\mathrm{W}^{\mathrm{ap}}}$, of the system can be calculated. However, in both limiting cases $c_{\mathrm{W}}$ ap is significantly lower than the actual water concentration in the system, $c_{\mathrm{W}}$. Apparently, some water molecules are immobilized by the hydrophilic interface (see above). The number of water molecules irrotationally bound by one DU molecule, $Z_{\mathrm{ib}}$, can be calculated (see eqn (5)):

$$
Z_{\mathrm{ib}}=\frac{c_{\mathrm{W}}-c_{\mathrm{w}}^{\mathrm{ap}}}{c_{\mathrm{DU}}}
$$

Likewise, the number of slow water molecules per DU molecule, $Z_{\text {slow }}$, is determined by:

$$
Z_{\text {slow }}=\frac{c_{2}}{c_{\mathrm{DU}}}
$$

Together, $Z=Z_{\mathrm{ib}}+Z_{\text {slow }}$ yields the number of water molecules per DU molecule that are strongly influenced by the amphiphile, so that their behavior deviates considerably from bulk water. The results for the Pn $3 m$ phase, $4 \leq Z \leq 8$, depending on the water content and on the $g$ value assumed, are plotted in Fig. 6. Similarly, $2 \leq Z \leq 3$ was obtained for $\mathrm{L}_{\alpha}$ (data not shown), which compares very favorably with the results of a previous NMR study, where a value of $Z \approx 3$ was deduced from the quadrupole splitting of the $\mathrm{O}-\mathrm{H}$ signal. ${ }^{30}$

As can be seen from Table 1, no significant variations can be observed for the relaxation times $\tau_{2}$ and $\tau_{3}$ of processes 2 and 3 . While the dynamics of the H-bond network do not depend on the water concentrations within only one phase, it should be kept in
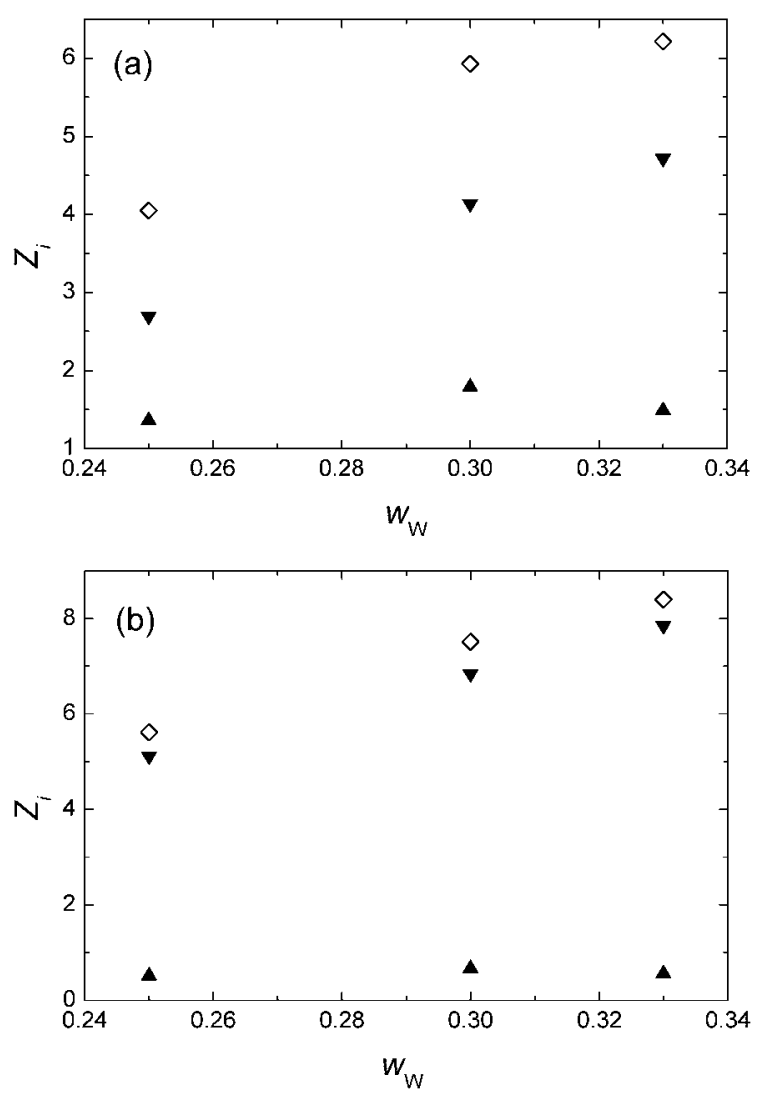

Fig. 6 Hydration numbers $Z_{\text {slow }}(\boldsymbol{\Delta}), Z_{\text {ib }}(\boldsymbol{\nabla})$ and $Z=Z_{\text {slow }}+Z_{\text {ib }}(\diamond)$ of $\mathrm{DU} / \mathrm{H}_{2} \mathrm{O}\left(P n 3 m, 35{ }^{\circ} \mathrm{C}\right)$ assuming two different values for the empirical orientational correlation factor, $g$ : (a) $g=1$; (b) $g=2.68$.

mind that, due to the narrow phase boundaries, only slight variations of the water content are possible in these binary mixtures.

\subsection{Ternary DUIR(+)-limonene/water mixtures}

Adding $R(+)$-limonene to DU/water mixtures leads to an increasingly negative curvature of the interface, which gives rise to a number of new phases (see Fig. 1), as has been previously outlined in detail. ${ }^{5}$ For our study, the hexagonal phase $\left(\mathrm{H}_{\text {II }}\right)$ is of particular interest, as its extension over the phase diagram allows variations in the water content over a wide range; thus, it is possible to observe the development of the relaxation parameters as the saturation line is approached. This is essential as the internal structure in fully hydrated monolinolein-water bulk samples (i.e. bulk samples at the saturation line, see Fig. 1) corresponds exactly to that of dispersed phases, as has been shown previously. ${ }^{5}$ The latter phases (which we call ISAsomes to emphasize that they are internally self-assembled) are very important for a number of applications; ${ }^{6 a-}$ unfortunately, it is not possible to study the behavior of the confined water directly in ISAsomes as there will always be interference with the excessive amount of outside bulk water present in those systems. However, the development of relaxation amplitudes $S_{i}$ and times $\tau_{i}$ when approaching the saturation line may provide some valuable information about the properties of the dispersed phase in ISAsomes.

Accordingly, two series of measurements have been performed at different DU/oil ratios $\delta=m_{\mathrm{DU}} /\left(m_{\mathrm{DU}}+m_{\mathrm{oil}}\right)$. In both cases 


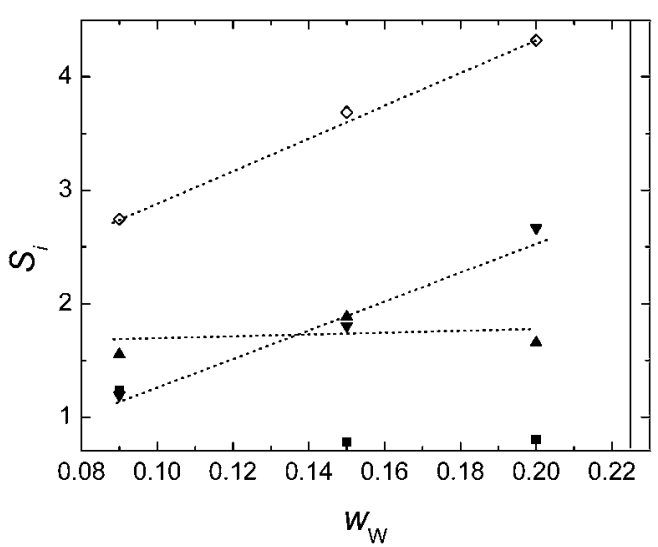

Fig. 7 Variation of relaxation amplitudes, $S_{i}$, of DU/R(+)-limonene/ $\mathrm{H}_{2} \mathrm{O}\left(\mathrm{H}_{\mathrm{II}}, 25^{\circ} \mathrm{C}, \delta=0.90\right)$ with water content, $w_{\mathrm{W}}: S_{1}(\boldsymbol{\square}), S_{2}(\boldsymbol{\Delta}), S_{3}$ $(\boldsymbol{\nabla})$ and $S_{2}+S_{3}(\diamond)$. The solid line indicates the saturation line; dashed and dotted straight lines are only included as a visual aid. (Similar results were obtained for $\delta=0.85$.)

( $\delta=0.85$ and $\delta=0.90)$, the slow-water amplitude $S_{2}$ seems to be almost independent of the water content, whereas the bulk-water amplitude $S_{3}$ increases (Fig. 7). Calculating $Z_{\text {ib }}$ and $Z_{\text {slow }}$ as outlined above, the values $1 \leq Z_{\mathrm{ib}} \leq 3(g=1)$ and $1.5 \leq Z_{\mathrm{ib}} \leq 4$ ( $g=2.68)$ are obtained, whereas $Z_{\text {slow }}$ remains constant at $\sim 1.0$
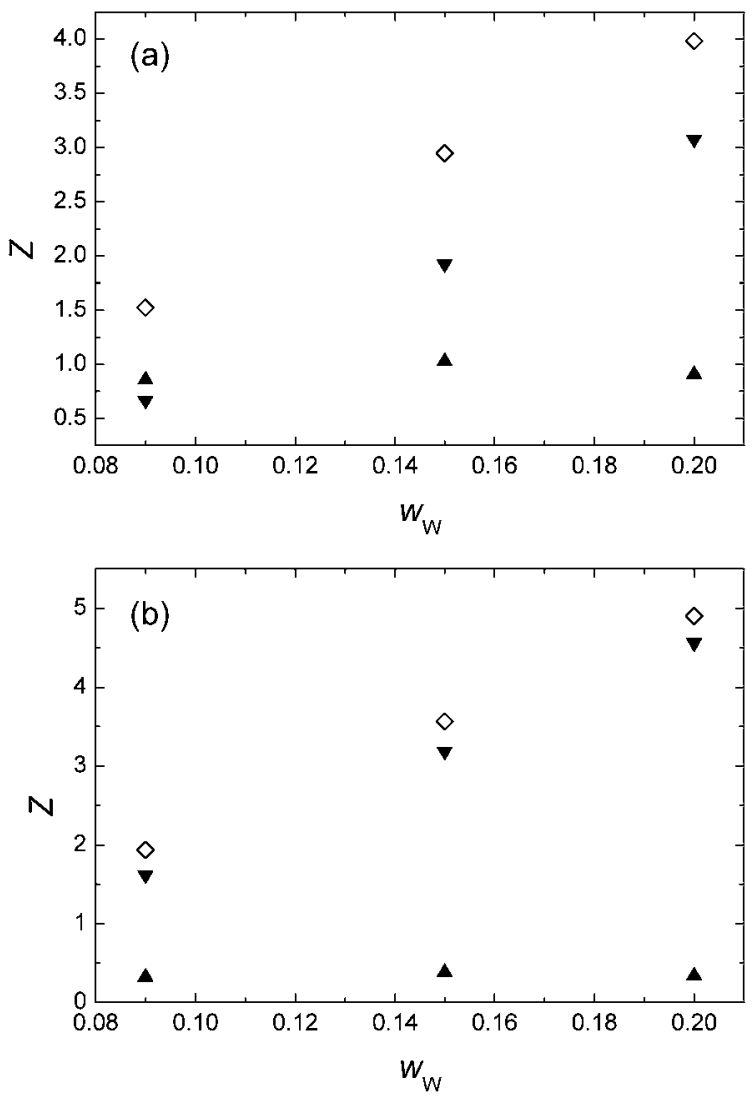

Fig. 8 Hydration numbers $Z_{\text {slow }}(\boldsymbol{\Delta}), Z_{\text {ib }}(\boldsymbol{\nabla})$ and $Z=Z_{\text {slow }}+Z_{\text {ib }}(\diamond)$ of DU/R(+)-limonene/ $\mathrm{H}_{2} \mathrm{O}\left(\mathrm{H}_{\mathrm{II}}, 25^{\circ} \mathrm{C}, \delta=0.90\right)$ assuming two different values for the empirical orientational correlation factor, $g$ : (a) $g=1$; (b) $g=2.68$.
( $g=1)$ and $\sim 0.5$ ( $g=2.68)$ (Fig. 8). Apparently, interfacial hydration increases considerably with increasing water content; however, only the number of immobilized water molecules per surfactant molecule increases, whereas the number of slow water molecules remains virtually constant. This trend can be seen even more clearly in Fig. 9 where the apparent concentrations of slow and bulk-like water ( $c_{2}{ }^{\text {ap }}$ and $c_{3}{ }^{\text {ap }}$ ), calculated according to eqn (4), are plotted as a fraction of the actual water concentration, $c_{\mathrm{W}}$, together with the bound-water contribution. Although it should be borne in mind that the true value of the orientational correlation factor $g$ is unknown (so that the absolute values should be regarded with caution), it is clear that the fraction of bound water strongly increases with increasing water content, whereas the percentage of slow water decreases and that of bulklike water remains roughly constant. In conclusion, a large fraction (at least 50\%, as can be seen from Fig. 9; note that for $g>$ 1 , even higher values are obtained) of the water molecules behave quite differently from bulk water near the saturation line. On the other hand, the relaxation times of the bulk-like process, $\tau_{3}$, approach the bulk-water value of 8.32 ps (ref. 23) when approaching the saturation line (Fig. 10). This means that near the saturation line, and hence also in the dispersed phase of ISAsomes, the bulk-like water does indeed share the dynamic properties of 'real' bulk water.
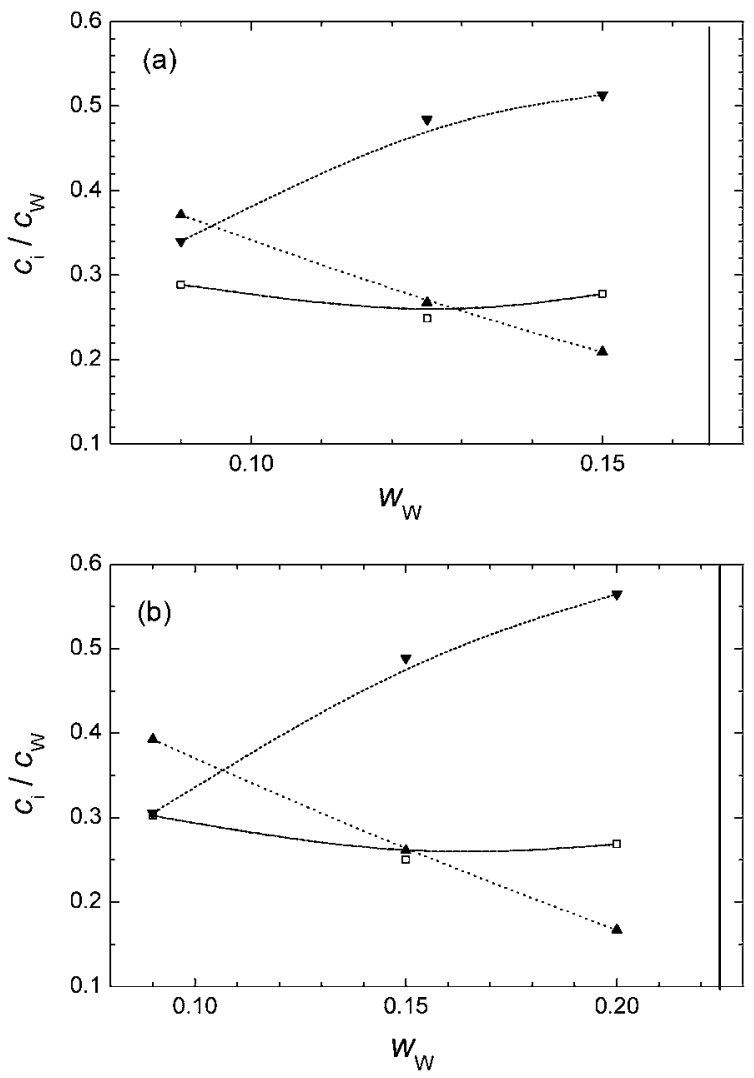

Fig. 9 Fractions of bulk-like $\left(\square, c_{3}{ }^{\text {ap }} / c_{\mathrm{W}}\right)$, slow $\left(\boldsymbol{\Lambda}, c_{2}{ }^{\text {ap }} / c_{\mathrm{W}}\right)$ and bound $\left(\mathbf{\nabla}, 1-c_{\mathrm{W}}{ }^{\text {ap }} / c_{\mathrm{W}}\right)$ water of $\mathrm{DU} / R(+)$-limonene $/ \mathrm{H}_{2} \mathrm{O}\left(\mathrm{H}_{\mathrm{II}}, 25^{\circ} \mathrm{C}\right.$, assuming $g=1$ ) for different water contents, $w_{\mathrm{W}}$ : (a) $\delta=0.85$; (b) $\delta=0.90$. Solid lines indicate the saturation line; dashed and dotted lines are only included as a visual aid. 


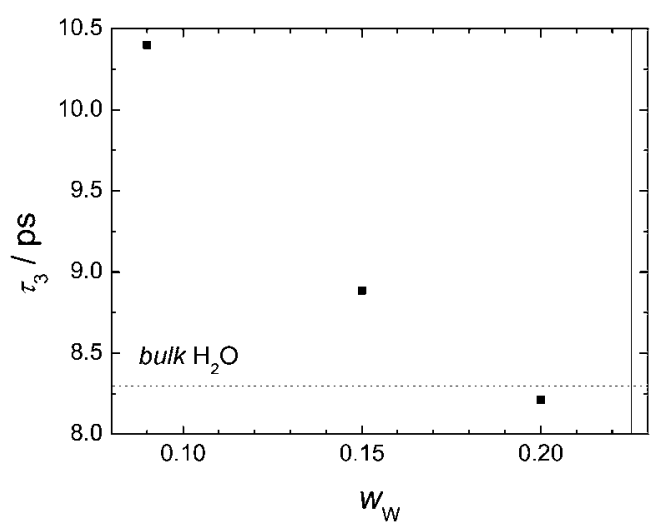

Fig. 10 Bulk-like water relaxation times $\tau_{3}(\mathbf{\square})$ of $\mathrm{DU} / R(+)$-limonene/ $\mathrm{H}_{2} \mathrm{O}\left(\mathrm{H}_{\mathrm{II}}, 25^{\circ} \mathrm{C}, \delta=0.90\right)$ when approaching the saturation line (solid). The bulk-water value, $\tau=8.32 \mathrm{ps}$ (ref. 23 ), is provided for comparison as a dotted line.

Differential scanning calorimetry. As Senatra et al. have previously shown, along with DRS, differential scanning calorimetry (DSC) is also able to distinguish between bound, interfacial and free water in W/O microemulsions and related systems. ${ }^{31}$ In addition, more recent studies have demonstrated that a comparison between these two complementary methods may provide valuable additional information about the properties of water in systems of interest. ${ }^{32}$ Accordingly, we used DSC to investigate samples of the $\mathrm{H}_{\text {II }}$ phase in this work as a qualitative complementary method to have an independent back-up for our DRS results.

Fig. 11 and 12 show the thermograms recorded for the two series of measurements at $\delta=0.85$ and $\delta=0.90$, respectively. At a first glance, two types of thermal events can be observed in each measurement, a small peak at approx. -8 to $-10{ }^{\circ} \mathrm{C}$ and a very broad peak at approx. -1 to $-3{ }^{\circ} \mathrm{C}$. For the samples exhibiting the highest water content $\left(w_{\mathrm{W}}=0.15\right.$ at $\delta=0.85$ and $w_{\mathrm{W}}=0.20$ at $\delta=0.90$ ), an additional peak at approx. $-1{ }^{\circ} \mathrm{C}$ is detected. According to the assignment made by Kogan et al. for bicontinuous microemulsions, peaks in these temperature ranges can be ascribed to 'bound', 'interphasal' and 'free' water, respectively. ${ }^{33}$ In principle, the existence of three different types of water confirms the DRS results presented above; however, the proportions of bound, interphasal (i.e. slow in the parlance of DRS) and free (bulk-like) water appear to be different. Clearly, the DSC signal ascribed to bound water is very low, whereas our DRS results suggest a substantial percentage of irrotationally bound water. Furthermore, bulk-like water, which yielded a considerable DRS signal for all samples, only appears in the DSC results for the highest water concentrations.

How can these apparent inconsistencies be explained? First of all, it should be remembered that the terms 'bound' or 'free' water are not general descriptions of absolute hydrogen-bond strengths, but are always associated with, and therefore highly dependent on a certain detection method. ${ }^{15}$ For example, when two water molecules are called 'irrotationally bound' in a DRS measurement, this means that the hydrogen bonds of both molecules are strong enough to prevent them from rotating on the DRS timescale. However, this does not necessarily imply that the binding strength of these two water molecules is equal.

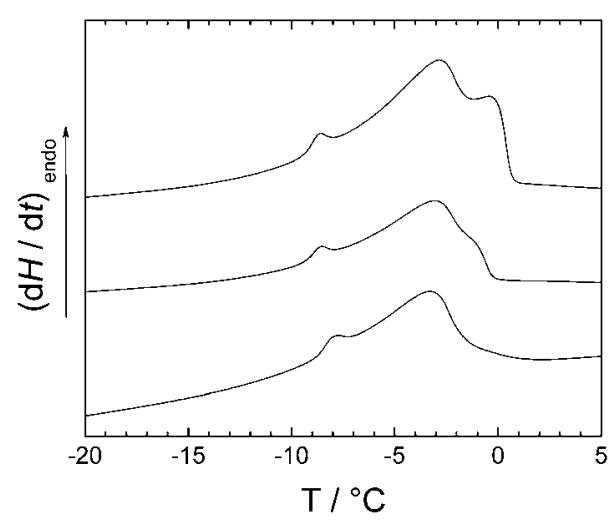

Fig. 11 Thermal behavior of DU/R(+)-limonene/ $\mathrm{H}_{2} \mathrm{O}\left(\mathrm{H}_{\mathrm{II}}, \delta=0.85\right)$ for various water contents, $w_{\mathrm{W}}=0.09,0.125$, and 0.15 (bottom to top).

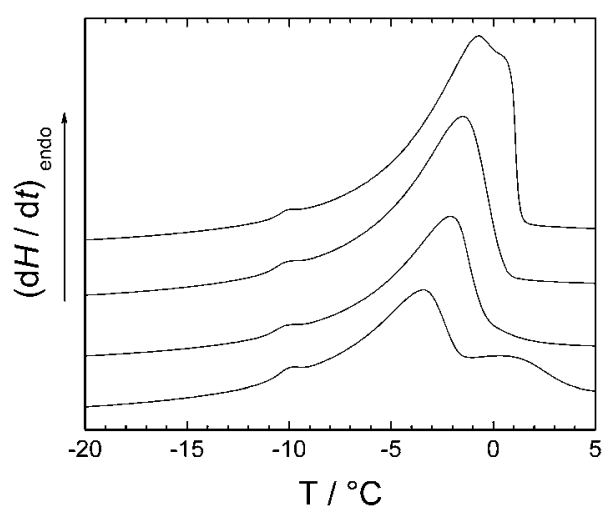

Fig. 12 Thermal behavior of $\mathrm{DU} / R(+)$-limonene $/ \mathrm{H}_{2} \mathrm{O}\left(\mathrm{H}_{\mathrm{II}}, \delta=0.90\right)$ for various water contents, $w_{\mathrm{W}}=0.09,0.125,0.15$, and 0.20 (bottom to top).

Hence, it is possible that one of them is bound just strongly enough not to move on the DRS timescale, but still contributes to the interphasal water peak in a DSC thermogram, while the second water molecule, being much more strongly bound, then contributes to the DSC bound-water peak.

Consequently, the very small bound water peak of the DSC thermograms can only partly account for the considerable levels of irrotationally bound water detected by DRS. On the other hand, the broad DSC interphasal water peak corresponds not only to the DRS slow water, but also to parts of the irrotationally bound and even to bulk-like water (except for the water-richest samples, where an additional free-water peak is observed in the DSC data). The latter appears plausible, given that the relaxation times of the DRS bulk-like water process 3 of $\sim 10$ ps are considerably slower than those of 'real' bulk water (see Fig. 10). They only reach the bulk-water value of $8.32 \mathrm{ps}$ (ref. 23) at $w_{\mathrm{W}}=$ 0.15 (for $\delta=0.85$ ) and at $w_{\mathrm{W}}=0.20$ (for $\delta=0.90$ ), when the additional free-water peak is detected in the DSC thermograms. Furthermore, the very broad shape of the interphasal water peak in all thermograms strongly suggests that it is composed of a number of different contributions, i.e. that it indicates the presence of several types of water influenced to various extents by the interface.

As a result, for weakly hydrated $\mathrm{H}_{\text {II }}$ phases, DRS and DSC detect different types of water, which are all influenced to varying 
degrees by the interface. However, when approaching the saturation line, both methods consistently show a certain fraction of water that behaves like bulk water. These findings also show that, in the internal aqueous phase of ISAsomes, a portion of the water molecules shares the dynamic properties of bulk water.

There is one more detail of these DSC results that merits attention. When comparing Fig. 11 and 12, it can be seen that the free-water signal is already apparent at a water content of $w_{\mathrm{W}}=$ 0.15 for $\delta=0.85$, but does not appear until $w_{\mathrm{W}}=0.20$ for $\delta=$ 0.90. In other words, with an increasing DU/limonene ratio, a larger proportion of the water is influenced by the interface. This implies that addition of limonene molecules renders the interface less 'effective' in binding water.

In principle, it is possible to determine the numbers $Z$ of water molecules per head group (as shown from the DRS results in Fig. 6 and 8) also from DSC experiments. However, our DSC data suffer from experimental problems during the measurements. The calibration of the instrument was not perfect and the actual data quality does not allow a reliable quantitative deconvolution of the endothermic overlapping water peaks. More precise DSC experiments are planned for the future.

\section{Conclusions}

In the first part of this study, both the inverse cubic $(P n 3 m)$ and the lamellar $\left(\mathrm{L}_{\alpha}\right)$ phases formed by the binary system DU/water were investigated by DRS. For all samples, three different 'types' of water were detected. The dielectric spectra exhibit two waterrelated relaxations. One of them (bulk-like water) is close to bulk water, albeit somewhat slowed down, and can obviously be ascribed to the water molecules far from the interface (i.e. in the interior of the inverse liquid-crystalline structures). The second relaxation (slow water) shows considerably reduced dynamics, which can be explained by interactions with the DU molecules at the interface. Furthermore, some water molecules are bound so strongly by the interface that they do not appear in the dielectric spectrum (irrotationally bound water). All three types of water represent considerable proportions of the total water content in the system. Water relaxation times do not depend significantly on the water concentration within one phase. However, due to the narrow phase boundaries, only slight variations in the water content are possible in binary DU/water mixtures.

In the second part of the study, the hexagonal $\left(\mathrm{H}_{\mathrm{II}}\right)$ phase of the ternary system $\mathrm{DU} / R(+)$-limonene/water was investigated. This phase is of special interest because it allows relatively large variations in water content, and thus, it is possible to track possible changes in water dynamics when approaching the saturation line. It has been shown previously that the latter corresponds to the internal structure of ISAsomes. Again, DRS reveals the three types of water described above. The interfacial hydration increases considerably with increasing water content; however, only the number of irrotationally bound water molecules per surfactant molecule increases, whereas the slow-water amplitude remains constant. The amplitude of the bulk-like water process also increases with increasing water content. Even more importantly, its relaxation is gradually accelerated until the bulk-water value of 8.32 ps (ref. 23) is reached when approaching the saturation line. This finding is supported by DSC measurements, where a small peak ascribed to bound water and a very broad peak interpreted as interphasal water are detected for all samples. However, when approaching the saturation line, an additional third peak emerges in the free-water region.

In conclusion, near the saturation line, and hence also in the dispersed phase of ISAsomes, at least $50 \%$ of the water molecules are strongly influenced by the interface, and therefore behave quite differently from bulk water. However, the remaining water does indeed share the dynamic properties of 'real' bulk water.

\section{Acknowledgements}

We would like to thank Dr Sandra Engelskirchen, Dr Karl Lohner and Angela Chemelli for stimulating discussions and Patricia Foditsch for her valuable support in the lab. We also thank the COST action D43 for partial financial support.

\section{References}

1 (a) J. Gustafsson, H. Ljusberg-Wahren, M. Almgren and K. Larsson, Cubic lipid-water phase dispersed into submicron particles, Langmuir, 1996, 12(20), 4611-4613; (b) J. Gustafsson, H. LjusbergWahren, M. Almgren and K. Larsson, Submicron particles of reversed lipid phases in water stabilized by a nonionic amphiphilic polymer, Langmuir, 1997, 13(26), 6964-6971; (c) K. Larsson, Colloidal dispersions of ordered lipid-water phases, J. Dispersion Sci. Technol., 1999, 20(1), 27-34.

2 A. Yaghmur, L. de Campo, L. Sagalowicz, M. E. Leser and O. Glatter, Emulsified microemulsions and oil-containing liquid crystalline phases, Langmuir, 2004, 21(2), 569-577.

3 P. T. Spicer, K. L. Hayden, M. L. Lynch, A. Ofori-Boateng and J. L. Burns, Novel process for producing cubic liquid crystalline nanoparticles (cubosomes), Langmuir, 2001, 17(19), 5748-5756.

$4 \mathrm{M}$. Caffrey, A lyotrope gradient method for liquid crystal temperature-composition-mesomorph diagram construction using time-resolved X-ray diffraction, Biophys. J., 1989, 55, 47-52.

5 A. Salonen, S. Guillot and O. Glatter, Determination of water content in internally self-assembled monoglyceride-based dispersions from the bulk phase, Langmuir, 2007, 23(18), 9151-9915.

6 (a) A. Yaghmur and O. Glatter, Characterization and potential applications of nanostructured aqueous dispersions, Adv. Colloid Interface Sci., 2009, 147-148, 333-342; (b) S. Guillot, M. Tomsic, L. Sagalowicz, M. E. Leser and O. Glatter, Internally selfassembled particles entrapped in thermoreversible hydrogels, $J$. Colloid Interface Sci., 2009, 330(1), 175-179; (c) B. J. Boyd, D. V. Whittaker, S.-M. Khoo and G. Davey, Lyotropic liquid crystalline phases formed from glycerate surfactants as sustained release drug delivery systems, Int. J. Pharm., 2006, 309(1-2), 218226; (d) M. Malmsten, Soft drug delivery systems, Soft Matter, 2006, 2, 760-769; (e) J. C. Shah, Y. Sadhale and D. M. Chilukuri, Cubic phase gels as drug delivery systems, Adv. Drug Delivery Rev., 2001, 47(2-3), 229-250.

7 J. Barthel, R. Buchner and M. Münsterer, Electrolyte Data Collection, Part 2: Dielectric Properties of Water and Aqueous Electrolyte Solutions, in Chemistry Data Series, ed. G. Kreysa, DECHEMA, Frankfurt am Main, 1995, vol. 12.

8 R. Buchner, J. Barthel and J. Stauber, The dielectric relaxation of water between $0{ }^{\circ} \mathrm{C}$ and $35^{\circ} \mathrm{C}$, Chem. Phys. Lett., 1999, 306(1-2), 57-63.

9 N. E. Levinger, Chemistry: water in confinement, Science, 2002, 298(5599), 1722-1723.

10 D. Cringus, J. Lindner, M. T. W. Milder, M. S. Pshenichnikov, P. Vöhringer and D. A. Wiersma, Femtosecond water dynamics in reverse-micellar nanodroplets, Chem. Phys. Lett., 2005, 408(1-3), $162-168$.

11 A. M. Dokter, S. Woutersen and H. J. Bakker, Ultrafast dynamics of water in cationic micelles, J. Chem. Phys., 2007, 126(12), 124507124510.

12 D. E. Rosenfeld and C. A. Schmuttenmaer, Dynamics of water confined within reverse micelles, J. Phys. Chem. B, 2006, 110(29), 14304-14312. 
13 N. Garti, A. Aserin, I. Tiunova and M. Fanun, A DSC study of water behavior in water-in-oil microemulsions stabilized by sucrose esters and butanol, Colloids Surf., A, 2000, 170(1), 1-18.

14 N. Garti, R. Feldenkriez, A. Aserin, S. Ezrahi and D. Shapira, Lubr. Eng., 1993, 49, 404- 411.

15 R. Buchner and G. Hefter, Interactions and dynamics in electrolyte solutions by dielectric spectroscopy, Phys. Chem. Chem. Phys., 2009, 11, 8984-8999.

16 F. Kremer and A. Schönhals, Broadband Dielectric Spectroscopy, Springer, Berlin, 2003.

$17 \mathrm{~W}$. Wachter, R. Buchner and G. Hefter, Hydration of tetraphenylphosphonium and tetraphenylborate ions by dielectric relaxation spectroscopy, J. Phys. Chem. B, 2006, 110(10), 51475154.

18 C. Baar, R. Buchner and W. Kunz, Dielectric relaxation of cationic surfactants in aqueous solution. 1. Solvent relaxation, J. Phys. Chem. B, 2001, 105(15), 2906-2913.

19 R. Mezzenga, C. Meyer, C. Servais, A. I. Romoscanu, L. Sagalowicz and R. C. Hayward, Shear rheology of lyotropic liquid crystals: a case study, Langmuir, 2005, 21(8), 3322-3333.

20 R. Buchner, G. T. Hefter and P. M. May, Dielectric relaxation of aqueous $\mathrm{NaCl}$ solutions, J. Phys. Chem. A, 1998, 103(1), 1-9.

21 R. Buchner, C. Hölzl, J. Stauber and J. Barthel, Dielectric spectroscopy of ion-pairing and hydration in aqueous tetra- $n$ alkylammonium halide solutions, Phys. Chem. Chem. Phys., 2002, 4(11), 2169-2179.

22 R. Buchner, T. Chen and G. Hefter, Complexity in "simple" electrolyte solutions: ion pairing in $\mathrm{MgSO}_{4}(\mathrm{aq}), J$. Phys. Chem. B, 2004, 108(7), 2365-2375.

23 T. Fukasawa, T. Sato, J. Watanabe, Y. Hama, W. Kunz and R. Buchner, Relation between dielectric and low-frequency Raman spectra of hydrogen-bond liquids, Phys. Rev. Lett., 2005, 95, 197802.

24 (a) M. V. Fedotova; S. E. Kruchinin; H. M. A. Rahman; R. Buchner, Features of ion hydration and association in aqueous rubidium fluoride solutions at ambient conditions. J. Mol. Liq., DOI: 10.1016/j.molliq.2010.04.009; (b) H. M. A. Rahman and R. Buchner, unpublished results; (c) A. Tromans, P. M. May, G. Hefter, T. Sato and R. Buchner, Ion pairing and solvent relaxation processes in aqueous solutions of sodium malonate and sodium succinate, J. Phys. Chem. B, 2004, 108(36), 13789-13795.

25 D. J. Tobias and M. L. Klein, Molecular dynamics simulations of a calcium carbonate/calcium sulfonate reverse micelle, J. Phys. Chem., 1996, 100(16), 6637-6648.

26 W. Wachter, Cooperative Dynamics of Didodecyldimethylammonium Bromide/Water/ $n$-Dodecane Microemulsions, A Dielectric Relaxation Study, Regensburg University, Regensburg, 2007.

27 U. Kaatze, R. Behrends and R. Pottel, Hydrogen network fluctuations and dielectric spectrometry of liquids, J. Non-Cryst. Solids, 2002, 305(1-3), 19-28.

28 R. Buchner, Dielectric Spectroscopy of Solutions, in Novel Approaches to the Structure and Dynamics of Liquids: Experiments, Theories and Simulations. NATO Science Ser. II, ed. J. Samios and V. A. Durov, Kluwer, Dordrecht, 2004, vol. 133.

29 J. Barthel, H. Hetzenauer and R. Buchner, Ber. Bunsen-Ges. Phys. Chem., 1992, 96, 1424-1432.

30 W. G. Morley and G. J. T. Tiddy, Phase behaviour of monoglyceride/ water systems, J. Chem. Soc., Faraday Trans., 1993, 89, 2823-2831.

31 D. Senatra, G. Gabrielli, G. Caminati and Z. Zhou, Conformational changes at the microemulsion water/oil interface and their influence on the system's dielectric temperature behavior, IEEE Trans. Electr. Insul., 1988, 23(4), 579-589.

32 S. Ezrahi, I. Nir, A. Aserin, N. Kozlovich, Y. Feldman and N. Garti, Dielectric and calorimetric characteristics of bound and free water in surfactant-based systems, J. Dispersion Sci. Technol., 2002, 23(1), 351-378.

33 A. Kogan, D. E. Shalev, U. Raviv, A. Aserin and N. Garti, Formation and characterization of ordered bicontinuous microemulsions, J. Phys. Chem. B, 2009, 113(31), 10669-10678. 